\title{
Micorrizas arbusculares asociadas al pasto angleton (Dichathium aristatum Benth) en fincas ganaderas del municipio de Tolú, Sucre-Colombia
}

\section{Arbuscular mycorrhizae associated to angleton grass (Dichathium aristatum Benth) on livestock farms at the municipality of Tolú, Sucre-Colombia}

\author{
Alexander Pérez C, ${ }^{1 *}$ Ph.D, Víctor Peroza $C_{1}{ }^{2}$ M.Sc.
}

\begin{abstract}
${ }^{1}$ Universidad de Sucre, Facultad de Ciencias Agropecuarias, Grupos de investigación en Bioprospección Agropecuaria, Campus universitario Puerta Roja, Sincelejo, Colombia. ${ }^{2}$ Universidad de Sucre, Facultad de Ciencias Agropecuaria.*Correspondencia: alexander.perez@unisucre.edu.co
\end{abstract}

Recibido: Julio de 2011; Aceptado: Febrero de 2012.

\section{RESUMEN}

Objetivo. El presente estudio consistió en caracterizar el porcentaje de colonización de hongos formadores de micorrizas arbusculares (HMA); asociados a la rizósfera del pasto Angleton (Dichanthium aristatum) Benth de fincas ganaderas del municipio de Tolú, departamento de Sucre, Colombia. Materiales y métodos. Se muestrearon en total 35 fincas ganaderas, de las cuales se tomaron 10 muestras de suelo con raíces por finca a una profundidad de $20 \mathrm{~cm}$. De cada muestra se aislaron esporas de HMA, se hizo separación de morfotipos y su respectiva identificación a través de claves taxonómicas. De las raíces colectadas se determinó el porcentaje de colonización utilizando métodos de coloración. Resultados. Los ANAVA multifactorial entre las variables densidad de esporas $/ 100 \mathrm{~g}$ de suelo y porcentaje de colonización en función a zonas y corregimiento mostraron diferencias altamente significativas. Los resultados obtenidos indican que el $92 \%$ de las especies encontradas corresponden a características de hongos HMA similares al género Glomus, el $4 \%$ al género Gigaspora y el $4 \%$ restante a Paraglomus. Conclusiones. Este estudio preliminar realizado en el Caribe colombiano muestra la diversidad, densidad poblacional y el porcentaje de colonización de HMA asociadas al pasto Angleton en condiciones de siete corregimientos pertenecientes a dos zonas agrológicas del municipio de Tolú, departamento de Sucre.

Palabras clave: Forraje, hongos, micorrizas, rizósfera (Fuente: DeCS). 


\begin{abstract}
Objective. The present study was carried out to isolate, identify and determine the colonization rate of arbuscular mycorrhizal fungi (AMF) associated with rhizosphere of Dichanthium aristatum Benth grass, in livestock farms of the municipality of Tolú, department of Sucre. Materials and methods. Soil samples and roots from the different farms were picked up. Of each sample AMF spores were isolated, morphotype separation and identification was done by taxonomic keys. From the collected roots colonization rate were measured and calculated through staining methods. Results. 35 livestock farms were sampled, distributed throughout 7 areas of the municipality. The multifactorial ANOVA between the flux density of spores per $100 \mathrm{~g}$ soil and colonization percentage in terms of areas and farm factors showed highly significant differences. The results indicate that $92 \%$ of the species found are characteristic of fungi similar to the genus Glomus AMF, $4 \%$ to the genus Gigaspora, and the remaining $4 \%$ to Paraglomus. Conclusions. This preliminary study carried out in the Colombian Caribbean shows diversity, population density and the percentage of colonization of HMA Associated to Angleton grass in conditions of seven townships belonging to two agrological areas of the municipality of Tolú, Department of Sucre.
\end{abstract}

Key words: Fiber, fungi, mycorrhizal, rhizosphere (Source: DeCS).

\section{INTRODUCCIÓN}

La subregión fisiográfica Golfo del Morrosquillo (Colombia), cuenta con un total de 200.300 ha, de las cuales 149.349 son aptas para pasturas es decir representan el $74.56 \%$ del área total de la subregión y el $23.8 \%$ del área total de pasto en el departamento (1).

Entre las principales especies de pasturas cultivadas en la región Caribe colombiana, se encuentran: Botriochloa pertusa (Colosoana o kikuyo); Branchiaria mutica (Admirable); Dichanthium aristatum (Angleton) y Pennisetum $s p$ (King grass). El pasto Angleton (Dichantium aristatum Benth) representa la tercera especie con mayor área sembrada en Sucre, alcanzando una extensión de aproximadamente 56.200 ha, distribuidas en 19 municipios. El municipio de Santiago de Tolú cuya principal actividad económica es la ganadería semi-intensiva cuenta con la segunda mayor área sembrada (9.400 ha) con esta especie en el departamento

Los suelos de las fincas ganaderas del municipio de Tolú, se caracterizan por tener topografía plana, sujetos a inundación, químicamente tienen de mediana a alta saturación de bases, su profundidad efectiva varía de superficial a moderada, limitada por fluctuaciones del nivel freático, presentan cantidades variables de sodio, niveles de fósforo de medio a alto, contenido de bajo a mediano materia orgánica, $\mathrm{pH}$ de ligeramente ácido a ligeramente alcalino y fertilidad de media a alta. Estos suelos son aptos para cultivos que toleren la presencia de sales de sodio y las inundaciones.

Los factores fisiográficos, las acciones antropogénicas degenerativas del medio ambiente y el empleo de tecnologías inadecuadas, han dado lugar a la degradación de propiedades físicas, químicas y biológicas del suelo, lo que limita la oferta y calidad de pastos y forrajes principalmente en temporada seca (3). La sequía y la salinidad son factores limitantes en la producción agrícola, los estudios sobre micorrizas en estos ambientes son relativamente recientes. Diversos autores señalan que la mejora de la nutrición fosforada en plantas micorrizadas es un factor clave que justifica el incremento de tolerancia a condiciones de salinidad (4).

La actividad microbiana en la rizósfera es en parte la responsable del funcionamiento de los ecosistemas terrestres y de la fertilidad de los suelos agrícolas. Entre los microorganismos benéficos claves de la rizósfera, están los hongos formadores de micorrizas arbusculares (HMA), contribuyendo con el desarrollo y la nutrición de la planta, como también con el incremento de la tolerancia de los cultivos frente a determinadas condiciones de estrés de tipo biótico o abiótico. La integración de estos microorganismos teniendo en cuenta además de los parámetros físico-químico del suelo y los climático en el sistemas garantizan la sostenibilidad, contribuyendo a optimizar la calidad y la salud del suelo, suministrar el aporte de nutrientes e incrementar los rendimientos (5).

Los HMA, son una asociación simbiótica entre los hongos del filo Glomeromycota y la raíz de la mayoría de las especies de plantas en el planeta. La asociación está caracterizada por un intercambio de nutrientes y la generación de diversos beneficios para la planta. En ella ocurre 
la formación de una extensa red de micelio en el suelo que les proporciona múltiples ventajas tales como: el mayor transporte de agua y nutrientes (especialmente $\mathrm{P}, \mathrm{Cu}$ y $\mathrm{Zn}$ entre otros); protección en condiciones de estrés, debido a problemas de salinidad, sequía, acidéz, elementos tóxicos o patógenos que atacan a la raíz (6). En la naturaleza, la mayor parte de las plantas presentan este tipo de asociación (7) y aunque aparentemente no existe especificidad taxonómica, evidencias recientes obtenidas con técnicas moleculares indican que las plantas son colonizadas preferentemente por ciertas especies de HMA con base en sus efectos diferenciales sobre el crecimiento vegetal.

Tradicionalmente la identificación y clasificación de los HMA ha venido realizándose de acuerdo a las características morfológicas de las esporas, el tamaño, color y número, grosor y consistencia de las paredes, así como la formación de la espora a partir de las hifas, que permite diferenciar familias, y la presencia o no de ornamentación, que permite diferenciar algunas especies. Con el advenimiento de los métodos moleculares, se ha logrado mayor diferenciación de los HMA; ocurriendo cambios en la sistemática de estos microorganismos como la reclasificación de los HMA del Phylum Zigomycota al Phylum Glomeromycota (8).

Algunos estudios han demostrado que el uso de HMA asociadas a plantas en condiciones de suelo salinos permite que estos vegetales se adapten mejor a las condiciones. Estudios recientes reportan que la inoculación de HMA incrementa la tolerancia de las plantas a la salinidad. Las micorrizas incrementan la tolerancia de las plantas al pastoreo por animales herbívoros, por un incremento en el suministro de nutrientes a las plantas hospedero, los cuales estimulan el rebrote continuo de las pasturas después de las defoliaciones hechas (9).

Teniendo en cuenta las bondades de esta simbiosis multifuncional natural con HMA y que en la actualidad a nivel de microbiología del suelo en la costa norte colombiana, no existen estudios sobre esta especie de pasto y su asociación con estos microorganismos, en condición de suelo salinos, el presente estudio tuvo como objetivo aislar los diferentes géneros de hongos formadores de micorrizas arbusculares nativas asociadas con la especie de pasto Angleton en el municipio de Tolú y su relación con las diferentes zonas agrológicas.

\section{MATERIALES Y MÉTODOS}

Localización. El muestreo se llevó a cabo en 35 fincas ganaderas establecidas con el pasto Angleton del municipio de Tolú, correspondiente a dos zonas agrológicas ubicadas según la clasificación de las tierras por su capacidad de uso en las subclases IIIsc y IVsc-Vhs (Tabla 1 ).

Tabla 1. Numero de corregimiento por zonas agrológicas muestreadas.

\begin{tabular}{ll}
\hline Corregimientos & Zonas agrologicas (subclases) \\
\hline Cabecera Municipal & Zona 1 (subclase IIIsc) \\
Puerto Viejo & Zona 1 (subclase IIIsc) \\
Santa Lucía & Zona 1 (subclase IIIsc) \\
Límites Coveñas & Zona 1 (subclase IIIsc) \\
Nueva Era & Zona 1 (subclase IIIsc) \\
Pita En Medio & Zona 1 (subclase IIIsc) \\
Pita Abajo & Zona 2 (subclase IVsc - Vhs) \\
\hline
\end{tabular}

El municipio de Tolú hace parte de la subregión llanura costera aluvial o del Golfo del Morrosquillo, se encuentra ubicado al noreste del departamento de Sucre, tiene una extensión de 35750 ha con $45 \mathrm{Km}$ de costa, ubicado a una altura que oscila entre 0 y $10 \mathrm{msnm}$, precipitación promedio anual de 900 a $1200 \mathrm{~mm}$. La estación de lluvias va desde finales de abril hasta finales de noviembre, con algunas disminuciones sustanciales entre junio y agosto, denominado veranillo de San Juan, la época seca tiene duración aproximada de 5 meses (diciembre - abril) presentándose los vientos más fuertes con predominio de las direcciones del norte y noreste. La actividad ganadera está concentrada en un relieve de llanura fluviomarina, caracterizada por extensiones planas con desniveles pequeños y pendientes suaves, menores del $3 \%$ y relieve de vallecitos que corresponden a áreas estrechas y alargadas en los cuales se depositan los materiales por aporte lateral o longitudinal.

Muestreo. En cada finca se tomaron aleatoriamente varias submuestras (suelo y raíces) a una profundidad $0-20 \mathrm{~cm}$ de las cuales se conformó una muestra de $2000 \mathrm{~g}$. Las muestras se depositaron en bolsas plásticas rotuladas con el número de la finca, corregimiento, área sembrada con Angleton y fecha de recolección (Tabla 1.). Una vez colectadas se refrigeraron hasta su utilización.

Procesamiento, aislamiento e identificación de morfotipos. Las muestras fueron tamizadas para separar suelos y raíces. Las muestras de suelo se utilizaron para realizar los siguientes análisis: aislamiento de las esporas de los HMA, conteo de esporas (esporas/100 g de suelo), 
identificación de morfotipos por forma, color y tamaño de las esporas, siguiendo la metodología reportada por Pérez y Vertel (10).

Las esporas de los diferentes morfotipos aislados, fueron depositadas en cajas de Petri y posteriormente se observaron las características similares a HMA. Una vez realizado el proceso anterior, se procedió a la identificación de morfotipos de HMA, mediante el uso de técnicas descriptivas por ICCS y VAMF (11).

Porcentaje de colonización. De las raíces separadas, se procedió a realizar un corte de aproximadamente $10 \mathrm{~cm}$ de longitud, las cuales fueron sometidas a técnicas de coloración. Después de la coloración, se colocaron paralelamente sobre láminas, se cubrieron con laminillas y se observaron con objetivo 40X, donde se realizó el conteo de 100 campos ordenadamente. En cada campo se determinó campos negativos y positivos. En los campos positivos se tuvo en cuenta el tipo de estructura presente (arbúsculos, vesículas, hifas y esporas). Los resultados obtenidos fueron expresados en porcentaje de colonización en raíces (10).

Análisis estadístico. Los resultados obtenidos de número de esporas y el porcentaje de colonización en función del corregimiento por zonas agrológicas, fueron correlacionados utilizando análisis de ANOVA multifactorial realizados en el programa estadístico $\mathrm{R}$ (12).

\section{RESULTADOS}

Densidad poblacional de esporas de micorrizas arbusculares. Los resultados del ANOVA multifactorial entre la variable número de esporas $/ 100 \mathrm{~g}$ de suelo (densidad poblacional o esporulación) en función de los corregimientos por zonas, mostró diferencias altamente significativas (Tabla 2). Para visualizar

Tabla 2. Anova multifactorial para densidad poblacional de HMA en función de corregimiento y zonas en el municipio de Tolú. zona 1 (C1: Cabecera Municipal, C2: Puerto Viejo, C3: Santa Lucía, C4: Límites Coveñas, C5: Nueva Era, C6: Pita En Medio. Zona 2(Pita abajo). C: corregimientos.

\begin{tabular}{lccccc}
\hline \multicolumn{1}{c}{ Fuente } & $\begin{array}{c}\text { Suma de } \\
\text { Cuadrados }\end{array}$ & GI & $\begin{array}{c}\text { Cuadrado } \\
\text { Medio }\end{array}$ & Razón-F & Valor-P \\
\hline EFECTOS & & & & & \\
PRINCIPALES & & & & & \\
A:Corregimientos & $1.12493 E 7$ & 5 & $2.24987 E 6$ & 20.66 & $0.0000 * * *$ \\
B:Zonas & $1.37309 E 7$ & 6 & $2.28849 E 6$ & 21.01 & $0.0000 * * *$ \\
RESIDUOS & $9.47423 E 6$ & 87 & 108899. & & \\
TOTAL & $3.06336 \mathrm{E} 7$ & 98 & & & \\
(CORREGIDO) & & & & & \\
\hline$* * *$. Altamente & & & & & \\
\end{tabular}

los resultados anteriores, se realizó un gráfico exploratorio en box plot (Figura 1). Los resultados de este gráfico muestran que el corregimiento 7 (Pita Abajo) localizado en la zona agrológica 2 presentó el mayor número de esporas $(1000 \pm 1800)$. Con respecto al corregimiento 3 (Santa Lucia) de la zona 1, donde se presentó el menor número de esporas $(500 \pm 650)$. En este mismos gráfico se observa que todos los suelos de las fincas pertenecientes al corregimiento 6 (Pita en medio), presentaron los rangos más

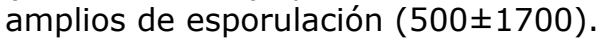

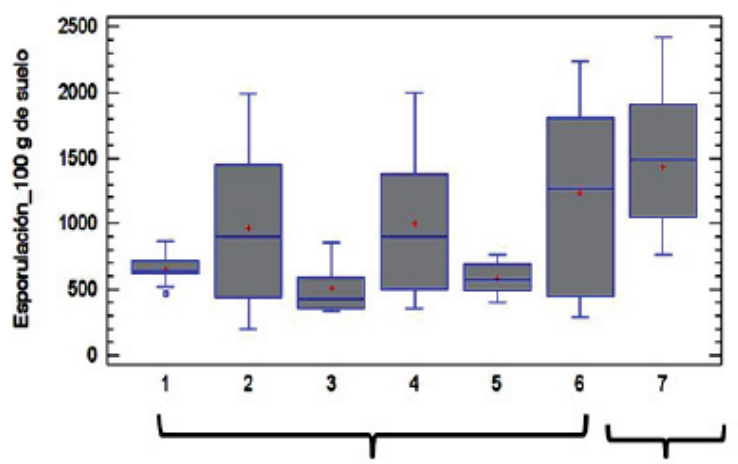

Corregimientos en zona 1 Corregimiento en zona 2

Figura1. Densidad de esporas/100 g de suelo de HMA en función de corregimientos y zonas agroecológicas con pasto angletón: zona 1 (C1: Cabecera Municipal, C2: Puerto Viejo, C3: Santa Lucía, C4: Límites Coveñas, C5: Nueva Era, C6: Pita En Medio. Zona 2(Pita abajo). C: corregimientos.

En condiciones químicas y de salinidad (Tabla 3) de los suelos de las diferentes fincas ganaderas, pertenecientes al corregimiento Pita Bajo de la zona 1 , en el momento del muestreo, se encontraron las mayores densidades de esporas (esporulación) de HMA, en relación al corregimiento 3 de la zona agrológica 1, donde se presentaron las menores cantidades de esporas.

Diversidad de HMA. En las 35 fincas ganaderas analizadas, se identificaron 25 morfotipos con características similares (cf) a HMA (Tabla 4). Fue observado que el morfotipo correspondiente al número 3 (Gigaspora albida Schenck \& Smith, 1982), morfotipo 14 (Glomus occultum Walker (1982)- Paraglomus occultum Morton \& Redecker, 2001) y morfotipo 25 (Glomus etunicatum Becker \& Gerdermann, 1977), fueron observados con mayor presencia en las diferentes muestras analizadas (datos no mostrados). Los resultados obtenidos señalan que el $92 \%$ de las especies encontradas correspondieron a características de hongos similares al género Glomus el $4 \%$ al género Gigaspora y el $4 \%$ restante a Paraglomus (Figura 2). Estos resultados evidencian que el género Glomus predomina y se adapta a las 
Tabla 3. Resultados de número de esporas (esporulación) y colonización de HMA, de acuerdo a análisis físicoquímico y de salinidad de los suelos.

\begin{tabular}{|c|c|c|c|c|c|c|c|c|c|c|c|c|c|c|}
\hline $\begin{array}{c}\text { Análisis } \\
\text { fiísico-químico }\end{array}$ & $\mathbf{p H}$ & м.O. & $\mathbf{P}$ & $\mathrm{Ca}$ & Mg & $\mathbf{K}$ & $\mathrm{Na}$ & CIC & $\mathrm{Cu}$ & Fe & Zn & Mn & Da & Corregimiento \\
\hline Esporulación & $\begin{array}{c}4.6 \\
\pm 6.25\end{array}$ & $\begin{array}{l}3.36 \\
\pm 5.8\end{array}$ & $\begin{array}{l}26.3 \\
\pm 87.5\end{array}$ & $\begin{array}{c}4.6 \\
\pm 21.9\end{array}$ & $\begin{array}{c}4.22 \\
\pm 13.44\end{array}$ & $\begin{array}{c}0.28 \\
\pm 0.56\end{array}$ & $\begin{array}{c}0.52 \\
\pm 1.01\end{array}$ & $\begin{array}{c}23.14 \\
\pm 33.61\end{array}$ & $\begin{array}{c}0.40 \\
\pm 1.60\end{array}$ & $\begin{array}{c}4.0 \\
\pm 51.60\end{array}$ & $\begin{array}{l}4.40 \\
\pm 9.6\end{array}$ & $\begin{array}{l}24.40 \\
\pm 62.8\end{array}$ & $\begin{array}{c}1.09 \\
\pm 1.63\end{array}$ & 7 (Pita abajo) \\
\hline \% de Colonización & $\begin{array}{c}5.05 \\
\pm 5.56 \\
\end{array}$ & $\begin{array}{l}3.36 \\
\pm 4.9\end{array}$ & $\begin{array}{c}33.6 \\
\pm 98.4\end{array}$ & $\begin{array}{c}8.0 \\
\pm 12.80\end{array}$ & $\begin{array}{c}4.24 \\
\pm 10.1\end{array}$ & $\begin{array}{c}0.2 \\
\pm 0.81\end{array}$ & $\begin{array}{c}0.40 \\
\pm 1.51\end{array}$ & $\begin{array}{c}17.26 \\
\pm 31.63\end{array}$ & $\begin{array}{c}0.80 \\
\pm 1.60\end{array}$ & $\begin{array}{c}20.4 \\
\pm 106.80 \\
\end{array}$ & $\begin{array}{l}4.40 \\
\pm 9.6 \\
\end{array}$ & $\begin{array}{c}35.60 \\
\pm 117.3\end{array}$ & $\begin{array}{c}1.38 \\
\pm 1.86 \\
\end{array}$ & 6 (Pita en medio) \\
\hline $\begin{array}{c}\text { Análisis de } \\
\text { salinidad }\end{array}$ & $\mathbf{p H}$ & CE & CIC & $\mathrm{Ca}$ & Mn & $\mathbf{K}$ & $\mathbf{N a}$ & $\mathrm{CaCO} 3$ & $\mathbf{C l}$ & SO4 & PSI & & & \\
\hline Esporulación & $\begin{array}{c}4.6 \\
\pm 6.25\end{array}$ & $\begin{array}{c}0.31 \\
\pm 0.90\end{array}$ & $\begin{array}{l}26.13 \\
\pm 27.5\end{array}$ & $\begin{array}{c}3.5 \\
\pm 28.0\end{array}$ & $\begin{array}{c}1.5 \\
\pm 24.17\end{array}$ & $\begin{array}{c}0.08 \\
\pm 0.88\end{array}$ & $\begin{array}{c}0.03 \\
\pm 0.05\end{array}$ & $\begin{array}{c}1.2 \\
\pm 4.0\end{array}$ & $\begin{array}{c}3.0 \\
\pm 58.0\end{array}$ & $\begin{array}{c}0.68 \\
\pm 10.42\end{array}$ & $\begin{array}{c}1.9 \\
\pm 9,99\end{array}$ & & & 7 (Pita abajo) \\
\hline \% de Colonización & $\begin{array}{c}5.05 \\
\pm 5.56\end{array}$ & $\begin{array}{c}0.32 \\
\pm 2.50\end{array}$ & $\begin{array}{c}17.36 \\
\pm 31.63\end{array}$ & $\begin{array}{l}7.5 \\
\pm 7.0\end{array}$ & $\begin{array}{c}2.4 \\
\pm 8.67\end{array}$ & $\begin{array}{c}0.02 \\
\pm 0.18\end{array}$ & 0.00 & $\begin{array}{l}2.8 \\
\pm 3.40\end{array}$ & $\begin{array}{c}1.2 \\
\pm 4.0\end{array}$ & $\begin{array}{c}1.20 \\
\pm 2.25\end{array}$ & $\begin{array}{c}2.04 \\
\pm 6.38\end{array}$ & & & 6 (Pita en medio) \\
\hline
\end{tabular}

Tabla 4. Morfotipos de HMA aislados de suelo rizosférico de pasto angletón, en finas ganaderas del municipio de Tolú

\begin{tabular}{|c|c|}
\hline $\begin{array}{l}\text { No de } \\
\text { morf. }\end{array}$ & Características similares (cf) \\
\hline 1 & Cf Glomus aggregatum Schenck \& Smith, 1985 (11) \\
\hline 2 & Cf Glomus multicaule Gerdemann \& Bakshi, 1976 (11) \\
\hline 3 & Cf Gigaspora albida Schenck \& Smith, 1982 (11) \\
\hline 4 & Cf Glomus claroideum Schenck \& Smith, 1982 (11) \\
\hline 5 & Cf Glomus ambisporum Smith \& Schenck, 1985. (11) \\
\hline 6 & Cf Glomus leptotichum Schenck \& Smith, 1982. (11) \\
\hline 7 & Cf Glomus fulvum Trappe \& Gerdemann, 1922 (11) \\
\hline 8 & Glomus sp. \\
\hline 9 & Cf Glomus boreale Trappe \& Gerdemann, 1922 (11) \\
\hline 10 & Glomus sp. \\
\hline 11 & Cf Glomus invermayanum Hall, 1977 (11) \\
\hline 12 & Cf Glomus maculosum Millar \& Walker, 1986 (11) \\
\hline 13 & Cf Glomus fecundisporum Schenck \& Smith, 1982 (11) \\
\hline 14 & $\begin{array}{l}\text { Cf Glomus occultum Walker (1982) Paraglomus occultum } \\
\text { Morton \& Redecker, } 2001 \text { (11) }\end{array}$ \\
\hline 15 & Cf Glomus fragilistratum Skou \& Jakobsen, 1989 (11) \\
\hline 16 & Cf Glomus geosporum Walker, 1982 (11) \\
\hline 17 & Cf Glomus dimorphicum Boyetchko \& Tewari, 1986. (11) \\
\hline 18 & Cf Glomus radiatum Trappe \& Gerdemann, 1974 (11) \\
\hline 19 & Cf Glomus fasciculatum Walter \& Koske, 1987 (11) \\
\hline 20 & Cf Glomus diaphanum, Morton \& Walker, 1984 (11) \\
\hline 21 & Cf Glomus trimulares Koske \& Halvorson, 1989. (11) \\
\hline 22 & Sclerocystis sp (Glomus sp Morton \& Redecker, 2001) (11) \\
\hline 23 & Cf Glomus clarum Nicolson \& Schenck, 1979 (11) \\
\hline 24 & Glomus sp. \\
\hline 25 & Cf Glomus etunicatum Becker \& Gerdermann, 1977. (11) \\
\hline
\end{tabular}

morf. = morfotipos

condiciones edafoclimáticas del municipio de Tolú establecidas con pasto Angleton.

En las figuras 3 y 4 se observan los 25 morfotipos de HMA encontrados e identificados mediante el uso de manuales taxonómicos para micorrizas, en los suelos de las fincas ganaderas estudiadas.

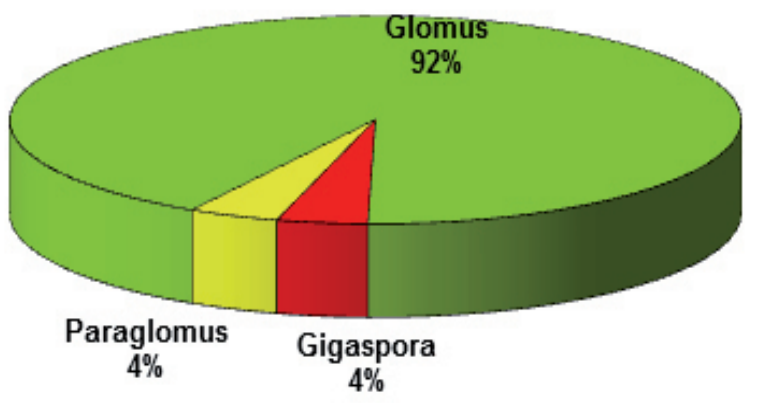

口Glomus $\quad$ Gigaspora $\quad$ Paraglomus

Figura 2. Distribución porcentual de géneros de hongos formadores de micorrizas arbusculares asociados al pasto angletón en los siete corregimientos del municipio de Tolú.

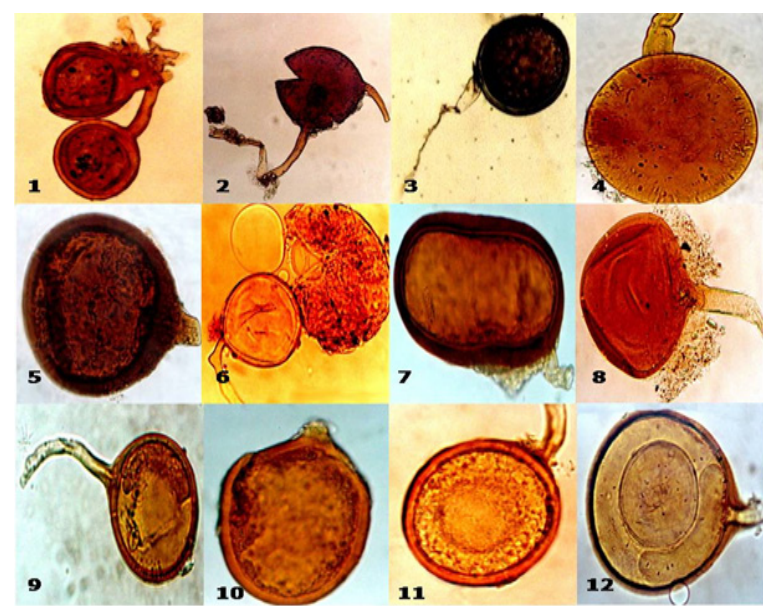

Figura 3. Morfotipos de hongos formadores de micorrizas arbusculares en rizósfera de suelo de fincas ganaderas del municipio de Tolú. 1-12: morfotipos de HMA identificados. Los números de los morfotipos corresponden a la clasificación realizada en la tabla 2 . Fuente: Peroza (2). 


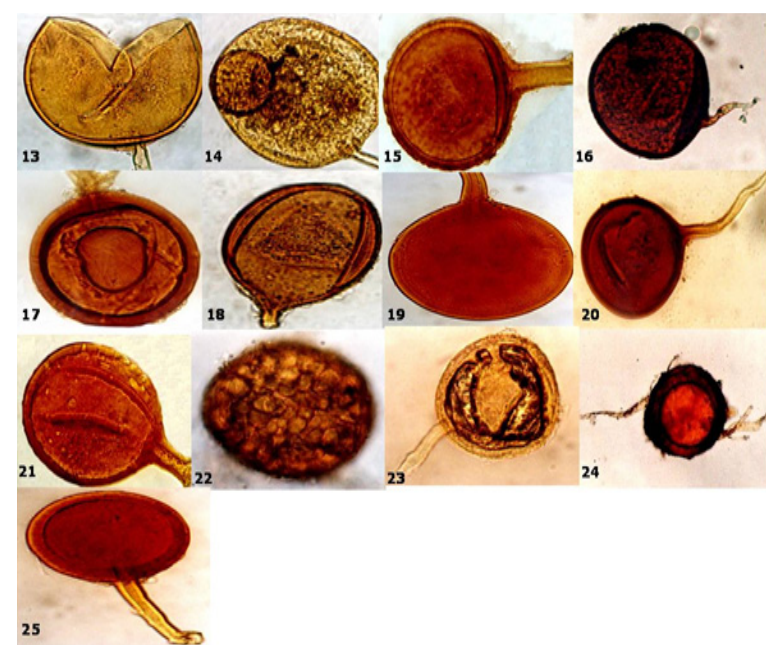

Figura 4. Morfotipos de hongos formadores de micorrizas arbusculares en rizósfera de suelo de fincas ganaderas del municipio de Tolú. 13-25: morfotipos de HMA identificados. Los números de los morfotipos corresponden a la clasificación realizada en la tabla 2. Fuente: Peroza (2).

Porcentaje de colonización en raíces. La tabla 5 , reporta los resultados obtenidos en el ANOVA multifactorial entre el porcentaje de colonización de HMA en raíces del pasto Angleton con relación a las variables corregimientos y zonas. Se observa además, que existen diferencias altamente significativas entre el porcentaje colonización y las variable corregimiento ( $p$-value: 0.0019) y zonas ( $p$-value: 0.0017). La figura 5 muestra las diferencias encontradas a través del gráfico exploratorio box plot entre colonización y las variables analizadas. El grafico señala que las fincas ubicadas en el corregimiento de Pita en Medio (6), presentaron los mayores

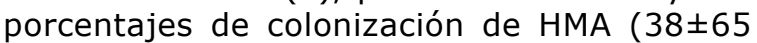
$\%)$ con respectos a las fincas del corregimiento Santa Lucia (3) en donde fueron encontrados los menores porcentajes de colonización $(20 \pm 38 \%)$.

Tabla 5. Anova multifactorial para el porcentaje de colonización de HMA en función a corregimiento y zonas en el municipio de Tolú. zona 1 (C1: Cabecera Municipal, C2: Puerto Viejo, C3: Santa Lucía, C4: Límites Coveñas, C5: Nueva Era, C6: Pita En Medio. Zona 2(Pita abajo). C: corregimientos.

\begin{tabular}{llllll}
\hline \multicolumn{1}{c}{ Fuente } & $\begin{array}{c}\text { Suma de } \\
\text { Cuadrados }\end{array}$ & GI & $\begin{array}{c}\text { Cuadrado } \\
\text { Medio }\end{array}$ & Razón-F & Valor-P \\
\hline EFECTOS & & & & & \\
PRINCIPALES & & & & & \\
A:Corregimientos & 3969.02 & 5 & 793.804 & 4.19 & $0.0019 * * *$ \\
B:Zonas & 4442.01 & 6 & 740.335 & 3.91 & $0.0017 * * *$ \\
RESIDUOS & 16487.9 & 87 & 189.516 & & \\
TOTAL & 23834.7 & 98 & & & \\
(CORREGIDO) & & & & & \\
\hline ***: Altamente significativa al $95.0 \%$ & de nivel de &
\end{tabular}

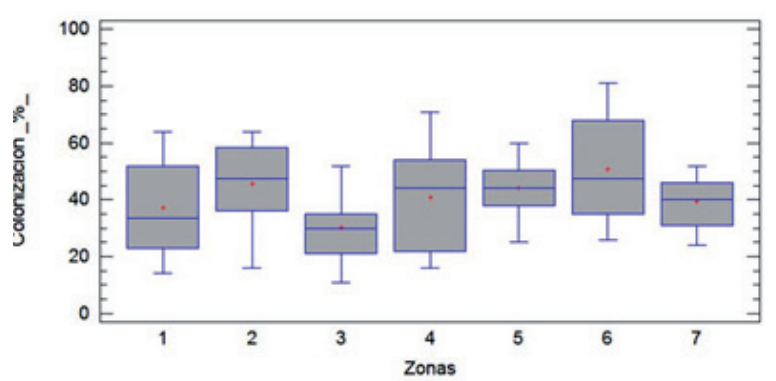

Figura 5. Porcentaje de colonización de hongos formadores de micorrizas arbusculares en raíces de Angleton (Dichanthium aristatum Benth) en función a corregimientos y zonas. zona 1 (C1: Cabecera Municipal, C2: Puerto Viejo, C3: Santa Lucía, C4: Límites Coveñas, C5: Nueva Era, C6: Pita En Medio. Zona 2(Pita abajo). C: corregimientos.

Los resultados mostrados en la tabla 3, indican que los mayores porcentajes de colonización observados en raíces del pasto Angleton fueron encontrados en las condiciones químicas y de salinidad de los suelos presentados por las diferentes fincas ganaderas, pertenecientes al corregimiento Pita en el momento del muestreo.

\section{DISCUSIÓN}

De los 25 aislamientos encontrados, el género Glomus mostró la mayor presencia en los suelos; estudios realizados (10) con la especie de pasto Colosoana en condiciones de edafoclimatica de los suelos de fincas ganaderas del municipio de Corozal también reportaron un alto número de especies del género Glomus, lo que indica que en el departamento de Sucre, este es el predominante.

Dentro del género Glomus, la especie de Glomus etunicatum mostró mayor presencia en las diferentes muestras analizadas en condiciones del municipio de Tolú. Estudios realizado en Brasil con el pasto Brachiaria decumbens, inoculado con la especie $G$. etunicatum, en suelos conteniendo diferentes niveles de cloruro de sodio, mostraron que la colonización y el número de esporas de G. etunicatum, no fue afectado por incrementos en los niveles de cloruro de sodio aplicados al suelo, lo que sugiere que podría ser una especie adaptada y resistente a esta condición (13). Otra especie con mayor abundancia correspondió a Gigaspora albida, aislada en los suelos de fincas ganaderas donde predominó la textura franco arenoso. También se observó mayor presencia de esporas de HMA del género Gigaspora en suelos de fincas ganaderas en el municipio de Tolú donde se reportó predomino de texturas arenosas (2). 
Estudios específicos sobre la presencia de HMA en suelos salinos en Colombia son escasos y poco conclusivos con relación a la influencia de parámetros físicos-químicos y la salinidad sobre el colonización in situ de HMA, asociados a la rizósfera y raíces del pasto Angleton. Lo anterior hace difícil la comparación de los resultados obtenidos en este estudio; sin embargo los resultados obtenidos son bajos para el número de esporas y porcentaje de colonización de HMA en raíces de Angleton para las fincas ubicadas en el corregimiento de Santa Lucia (3) en las condiciones físico-químicas y de salinidad presentados en el momento del muestreo y los mayores valores para número de espora y porcentajes de colonización se encontraron en el corregimiento de Pita Abajo (7) y Pita en Medio (6), respectivamente.

Las fincas ubicadas en el corregimiento de Santa Lucía, presentaron de acuerdo a los resultados de los análisis físicos-químicos de los suelos, $\mathrm{pH}$ de muy ácido a fuertemente ácido, valores moderados a adecuado de materia orgánica, fósforo de alto a muy alto, valores muy altos de hierro, cobre zinc y manganeso y valores bajos a muy alto de calcio, contenidos adecuados a valores altos de potasio y magnesio y contenidos moderados de sodio. Los análisis de salinidad de los suelos mostraron valores de $\mathrm{pH}$ de medianamente ácido a ligeramente básico, valores de conductividad eléctrica (CE) no salino y salinidad del suelo de acuerdo con el porcentaje de sodio intercambiable (PSI) y la CE normal.

El corregimiento de Pita Abajo presentó los mayores porcentajes de colonización en raíz; mientras que el de Pita en Medio mostró las mayores densidades de esporas en el suelo, presentando las siguientes características físico-químicas y de salinidad, respectivamente: $\mathrm{pH}$ de muy fuertemente ácido a fuertemente ácido, contenidos moderados a adecuado de materia orgánica, fósforo de contenidos adecuados a alto, valores muy altos de hierro, cobre y zinc, contenidos moderados a alto de calcio, magnesio y potasio, sodio de contenidos moderados a altos.

Los análisis de salinidad de los suelos mostraron valores de $\mathrm{pH}$ de fuertemente ácido a ligeramente básico, valores de conductividad eléctrica (CE) no salino y salinidad del suelo de acuerdo porcentaje de sodio intercambiable (PSI) y la CE normal para la zona 6 y para la zona 7, $\mathrm{pH}$ de muy fuertemente ácido a ligeramente ácido, contenidos moderados a adecuado de materia orgánica, fósforo de contenidos adecuados a alto, valores muy alto de hierro, cobre zinc y manganeso y valores deficientes a muy alto de calcio, contenidos adecuados a valores altos de potasio y magnesio y sodio de contenidos moderados a altos. Los análisis de salinidad de los suelos mostraron valores de $\mathrm{pH}$ de fuertemente ácido a medianamente alcalino, valores de conductividad eléctrica (CE) no salino y salinidad del suelo de acuerdo al porcentaje de sodio intercambiable (PSI) y la CE normal.

Modelos matemáticos construidos para evaluar la densidad poblacional de esporas de HMA en suelos de fincas ganaderas del municipio de Tolú en función de parámetros físicos-químicos, indican que el mayor número de esporas de HMA en los suelos, está directamente relacionado con la concentración de los elementos intercambiables como magnesio $(\mathrm{Mg})$, sodio $(\mathrm{Na})$ y de valores de densidad aparente (Da) e inversamente relacionado con los valores de Cobre (Cu) y Manganeso (Mn) y los modelos construidos para predecir el número de esporas en función de la salinidad, muestra que esta interfiere directamente con la esporulación en presencia de las variables $\mathrm{pH}$, magnesio $(\mathrm{Mg})$, sodio $(\mathrm{Na})$, sulfato $\left(\mathrm{SO}_{4}\right)$, porcentaje de sodio intercambiable (PSI) y el porcentaje de sodio en partes por mil $\left(\mathrm{S} /{ }_{00}\right)$ e inversamente proporcional a la conductividad eléctrica (CEE) (14).

Modelos matemáticos construidos para predecir la colonización de HMA en raíces de Angleton, señalan que las variables que están directamente relacionadas son los valores de los elementos intercambiables de potasio $(\mathrm{K})$ y sodio ( $\mathrm{Na}$ ), de zinc $(Z n)$ y del porcentaje de hidrógeno en los suelos y son inversamente proporcionales a los valores de los elementos de $\mathrm{Ca}$ y $\mathrm{Mg}$ intercambiable. De otra parte se encontró mediante modelos matemáticos que la salinidad interfiere con la colonización de HMA en raíces de Angleton, en presencia del elemento calcio intercambiable y de los valores de PSI e inversamente a los valores del elemento Magnesio intercambiables y de bicarbonato $\left(\mathrm{HCO}_{3}\right)(14)$.

Estudios realizados en Colombia sobre el establecimiento de los HMA en condiciones naturales han demostrado que estos procesos biológicos están determinados por diversas condiciones tales como: factores físico-químicos del suelo ( $\mathrm{pH}$, contenido de fósforo, temperatura, aireación, textura y contenido de materia orgánica), condiciones climáticas (intensidad y duración de la luz, temperaturas, humedad, épocas de lluvias y épocas secas) y por las prácticas agronómicas (preparación del terreno, aplicación de pesticidas y prácticas culturales) (10). Sin embargo, aun faltan más estudios que permitan evaluar in situ, la esporulación de hongos formadores de micorrizas arbusculares asociadas a pasturas de importancia económica para la Región Caribe colombiana en diferentes agroecosistemas y en condiciones de salinidad. 


\section{REFERENCIAS}

1. Aguilera MM. Documento de trabajo sobre economía regional: La Economía del departamento de Sucre: Ganadería y Sector Público. Cartagena de India: Banco de la República; 2005.

2. Peroza CV. Caracterización de hongos formadores de micorrizas arbusculares (H.M.A.) nativas, asociadas con el pasto Angleton (Dichanthium aristatum Benth) en el municipio Tolú Departamento de Sucre. [Tesis de Maestría]. Bogotá, Colombia: Universidad Nacional de Colombia, Facultad de Agronomía, 2003.

3. Pérez $C R$, Pérez $C A$, Vertel $M M$. Caracterización nutricional, físico-química y microbiológica de tres abonos orgánicos para uso en agroecosistemas de pasturas en la subregión Sabanas del departamento de Sucre, Colombia. Rev Tumbaga 2010; 1:27-37.

4. Dodd JC. The role of arbuscular mycorrhizal fungi in agro-and natural ecosystems. Outlook Agric 2000; 29(1):55-62.

5. Barrer SE. El uso de hongos micorrízicos arbusculares como una alternativa para la agricultura. Rev Fac Cienc Agrop 2009; 7(1):123-133.

6. Jeffries $P$, Gianinazzi S, Perotto S, Turnau $\mathrm{K}$, Barea JM. The contribution of arbuscular mycorrhizal fungi in sustainable maintenance of plant health and soil fertility. Biol Fertil Soils 2003; 37(1):1-16.

7. Kapoor R, Sharma D, Bhatnagar AK. Arbuscular mycorrhizae in micropropagation systems and their potential applications. Sci Hortic 2008; 116:227-239.
8. Walker C, Schüßler A. Nomenclatural clarifications and new taxa in the Glomeromycota. Mycol Res 2004; 108(9): 981-982.

9. Ahn-Heum E, Gail W, Hartnett D. Effect of ungulate grazer on arbuscular mycorrhizal simbioses and fungal community structure in tall grass prairie. Mycol 2001; 93(2):233-242.

10. Pérez CA, Vertel MM. Evaluación de la colonización de micorrizas arbusculares en pasto Bothriochloa pertusa (L) A. Camus. Rev MVZ Córdoba 2010; 15(3):2165-2174.

11. International Culture Collection of Arbuscular \& Vesicular- Arbuscular Mycorrhizal Fungi. (INVAM, by Morton J B., Curator, Good for Basic Research), 2006. URL disponible en: http://infotree.library.ohiou.edu/scripts/ redirect.html?id=1661.

12. R Development Core Team, R: A language and environment for statistical computing, R Foundation for Statistical Computing, 2009, Vienna, Austria. ISBN 3-900051. URL disponible en: http://spatial-analyst.net/ book/biblio/author/650.

13. Mergulhao A, Buruty HA, Tabosa JN, Figueiredo MV, Maia LC. Salt stress response of Brachiaria plants with and without inoculation of rbuscular mycorrhizal fungi. Agrochim 2001; 45(1-2):24-31.

14. Peroza CV, Pérez CA. Efecto de parámetros físicos, químico y salinidad sobre la densidad poblacional y la colonización de micorrizas arbusculares en pasto Angleton en el municipio de Tolú, Sucre, Colombia. Rev Colombiana Cienc Anim 2010; 2(2):310-324. 